\title{
LA DÉHISCENCE DU CANAL SEMI CIRCULAIRE SUPÉRIEUR
}

S. KHARRAT, I. DRIDI, R. BOULAKBECH, C. EL AOUD, S. TABABI, R. ZAININE, N. BELTAIEF, E. MENIF*, S. SAHTOUT, G. BESBES.

\author{
SERVICE ORL ET CHIRURGIE MAXILLOFACIALE. \\ *SERVICE DE RADIOLOGIE \\ HÔPITAL LA RABTA. TUNIS.
}

\begin{abstract}
Introduction : La déhiscence du canal semi circulaire supérieur (CSCS ) est une entité rare de description récente dont la symptomatologie clinique typique est représentée par des vertiges déclenchés par des variations pressionnelles.

Matériel et méthodes: nous rapportons deux cas de déhiscence du CSCS ont été diagnostiqués grâce au scanner du rocher réalisé systématiquement devant des surdités mixtes à tympan normal.

Résultats : la déhiscence du CSCS était bilatérale dans 1 cas. Les 2 cas ont été révélés par une surdité mixte à tympan normal sans notion de vertiges. Le scanner ultra haute résolution des rochers en coupes coronales et sagittales a permis le diagnostic.
\end{abstract}

Conclusion : une déhiscence du CSCS doit être évoquée devant toute surdité de transmission ou mixte à tympan normal. Le scanner en coupes coronales et sagittales inframillimétriques permet le diagnostic.

Mots clés : canal semi circulaire supérieur, déhiscence, surdité.

\section{SUMMARY}

Introduction : Superior semicircular canal dehiscence is a rare entity witch is recently described. The common symptom is pressure-induced vertigo.

Patients and methods: we report 2 cases of superior semicircular canal dehiscence identified by computed tomography (CT) of the petrous bone in patients with mixed hearing loss and a normal tympanic membrane.

Results: Superior semicircular canal dehiscence was bilateral in 1 patient.

Mixed hearing loss with a normal tympanic membrane without vertigo was the presenting manifestation in all cases. Ultrahigh-resolution CT of the petrous bones with coronal and sagittal sections provided the diagnosis of superior semicircular canal dehiscence.

Conclusion: Mixed hearing loss with a normal tympanic membrane should suggest superior semi circular canal dehiscence. Coronal and sagittal CT sections less than $1 \mathrm{~mm}$ in thickness establish the diagnosis.

Key words : Superior semicircular canal, dehiscence, hearing loss.

\section{INTRODUCTION}

Le syndrome de déhiscence du canal semi circulaire supérieur est l'ensemble des phénomènes cliniques vestibulaires et cochléaires liés à la présence d'un défaut de couverture osseuse du toit du canal semi circulaire supérieur (ou antérieur). Il a été décrit pour la première fois par Minor en 1998 (1). Les manifestations cliniques typiques sont représentées par des vertiges induits par les variations de pression ou par l'exposition aux sons de forte intensité (phénomène de Tullio). Par ailleurs, une surdité de transmission ou mixte peut résumer le tableau clinique. Le scanner du rocher est l'examen paraclinique clé du diagnostic.

\section{OBSERVATIONS \\ Observation 1}

Patient âgé de 67 ans, sans antécédents pathologiques notables en particulier pas de notion de traumatisme, nous a consulté devant l'apparition d'une hypoacousie gauche d'aggravation progressive évoluant depuis 5 ans, associée à des acouphènes homolatéraux sans notion de vertiges induits par les variations pressionnelles, ni aux sons de forte intensité.

A l'otoscopie, les tympans étaient complets et normaux. L'examen neuro-vestibulaire était normal. Une audiométrie tonale a été réalisée objectivant une surdité mixte à $70 \mathrm{~dB}$ du côté gauche avec un Rinne moyen à $30 \mathrm{~dB}$. L'audition à droite était normale. A l'impédancemétrie, le tympanogramme était central avec un réflexe stapédien absent à gauche.

Le patient a bénéficié d'un scanner des rochers montrant une absence de couverture osseuse du canal semi circulaire supérieur (figures 1, 2). L'abstention thérapeutique a été décidée devant l'absence de symptomatologie vertigineuse invalidante. 


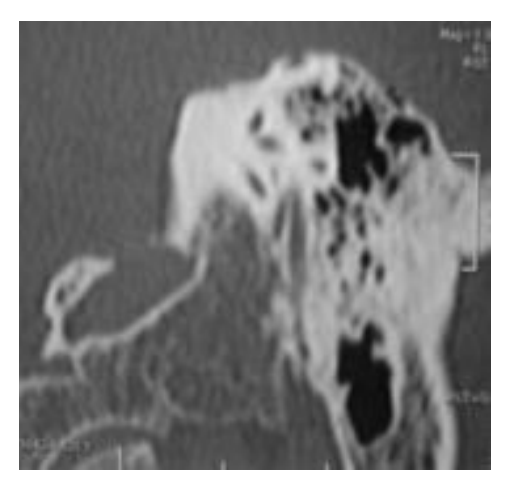

Fig. 1 : TDM des rochers en reconstruction sagittale : déhiscence du canal semi circulaire supérieur (flèche noire).

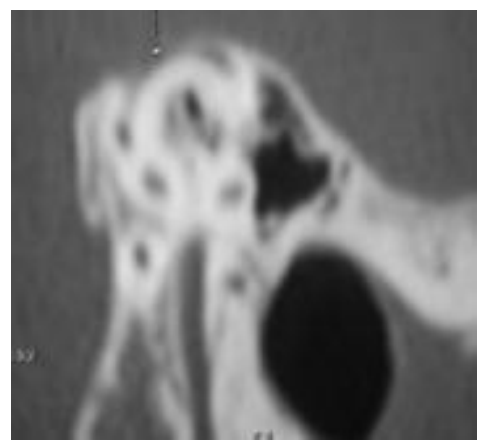

Fig. 2 : TDM des rochers, reconstruction dans l'axe du canal semi circulaire supérieur : absence de coque osseuse au niveau de son sommet (trait en pointillé)

\section{Observation 2}

Madame S, âgée de 42 ans sans antécédents pathologiques notables nous a consulté devant la notion d'hypoacousie bilatérale prédominant du côté droit, associée à des acouphènes bilatéraux sans notion de vertige.

A l'examen otoscopique, les tympans étaient complets et normaux. Les examens vestibulaire, neurologique et somatique étaient sans anomalies.

L'audiométrie tonale a montré une surdité mixte à droite à $70 \mathrm{~dB}$ avec un Rinne moyen à $40 \mathrm{~dB}$ et une surdité de transmission gauche à $30 \mathrm{~dB}$. A l'impédencemétrie, le tympanogramme était central avec des réflexes absents des deux côtés.

Un scanner des rochers a été pratiqué objectivant une déhiscence du canal semi-circulaire supérieur gauche (figure $n^{\circ} 3$ ). Une abstention thérapeutique a été préconisée devant l'absence de symptomatologie vertigineuse.

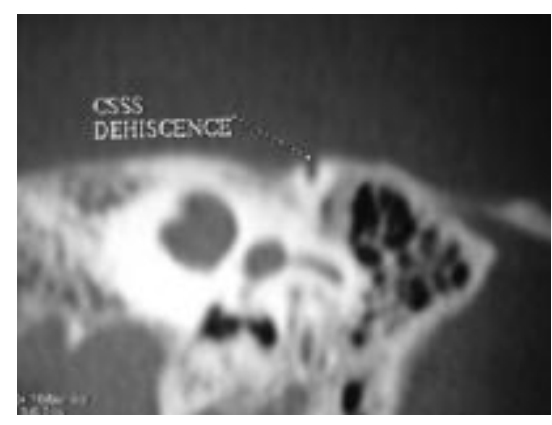

Fig. 3 : TDM des rochers en reconstruction sagittale : déhiscence du canal semi circulaire supérieur (trait en pointillé).

\section{DISCUSSION}

La déhiscence du canal semi circulaire supérieur (CSCS) est une entité rare de description récente $(1,2)$. La prévalence, selon une étude autopsique, serait de 0,67\% (13).

La déhiscence du CSCS a été décrite chez des patients âgés de 13 à 78 ans et semble être plus fréquente chez l'homme (2, 4, 5).

La déhiscence du CSCS est parfois d'origine post traumatique, le traumatisme inaugurant la symptomatologie $(2,4)$.

Elle pourrait être secondaire à une anomalie de développement osseux dans les premières semaines de vie et deviendrait symptomatique suite à un évènement déclenchant, soit un traumatisme minime ou une variation pressionnelle (3).

Les déhiscences du CSCS peuvent être unilatérales ou rarement bilatérales. Le taux de bilatéralité varie selon les séries entre $23,5 \%$ et $37,5 \%(5,6)$.

La topographie de la déhiscence est variable : le sommet du CSCS, la partie postérieure du CSCS au niveau du sinus pétreux supérieur, la partie antérieure du CSCS (7, 8). Chez nos deux patients, la déhiscence siégeait au niveau du sommet du canal semi-circulaire supérieur.

La symptomatologie clinique est variable et dépendrait de la taille de la déhiscence et de sa topographie $(9,10)$. Les vertiges et les oscillopsies induits par les variations de pression ou par les sons de forte intensité sont les symptômes cliniques les plus typiques $(11,12,13)$. L'examen clinique doit rechercher un nystagmus vertical torsionnel, induit par des variations de pression : manœuvre de Valsava, effort à glotte fermée, tympanométrie induisant une variation de pression dans le conduit auditif externe. On doit rechercher aussi ce nystagmus suite à une exposition à un son d'une intensité de 100 à 110 Décibel et à une fréquence de 500 à $2000 \mathrm{HZ}$.

La symptomatologie clinique peut consister en une surdité de transmission ou mixte $(1,2,3)$. La baisse de la conduction osseuse peut concerner uniquement les fréquences aigues ou apparaître dès les fréquences graves. 
Les réflexes stapédiens sont généralement présents. Cependant, dans la série de Mikulec (4), parmi huit patients présentant une déhiscence du CSCS, trois avaient une abolition des reflexes stapédiens, comme c'est le cas de nos patients. Des acouphènes synchrones au pouls sont parfois notés. Toutefois, cette déhiscence du CSCS peut être asymptomatique.

La surdité de transmission dans la déhiscence du canal semi circulaire supérieur s'expliquerait par la création d'une troisième fenêtre au niveau de l'oreille interne, qui va aboutir à une perte de l'énergie acoustique, d'où une augmentation de seuil de la conduction aérienne et ce qui entrainerait en parallèle un abaissement de seuil de la conduction osseuse en augmentant la différence d'impédance entre la fenêtre ronde et ovale (13-15).

Ce mécanisme de troisième fenêtre est également impliqué dans d'autres anomalies de l'oreille interne tel que le syndrome de l'aqueduc du vestibule large et les fistules labyrinthiques $(13,15)$.

Le phénomène de Tullio, est basé sur la réponse physiologique du vestibule à des stimuli sonores. D'après des expériences réalisées chez les chinchillas, la fenestration du CSCS abaisse le seuil de stimulation acoustique du vestibule et augmente l'amplitude des réponses (13). II a été démontré aussi qu'elle engendrait une hyperexcitabilité lors des variations pressionnelles.

Le potentiel évoqué otolithique teste le réflexe vestibulospinal. En cas de déhiscence du CSCS, on observe une augmentation de l'amplitude des ondes P13 et N23 (6). La tomodensitométrie de haute résolution (coupes de $1 \mathrm{~mm}$ ) ou ultra haute résolution (coupes de 0,5 ou $0,6 \mathrm{~mm}$ ) est l'examen clé du diagnostic. II faut cependant se méfier de fausses images de déhiscence du CSCS liées à une finesse du toit (inférieur à 0,5) $(9,10)$.
L'IRM n'est pas indiquée pour le diagnostic et est dans la majorité des cas interprétée comme normale (10).

Le traitement chirurgical est indiqué en cas de pathologie vestibulaire invalidante. Dans notre série, une abstention chirurgicale a été préconisée vue l'absence de symptomatologie vertigineuse.

L'intervention est une chirurgie de colmatage de la déhiscence. Elle consiste en une obturation de la fenestration à partir d'un abord de la fosse cérébrale moyenne car c'est le seul moyen d'objectiver la déhiscence. Un abord par voie transmastoïdienne a été proposé par plusieurs auteurs $(7,8,15)$.

Les matériaux de colmatage utilisés sont variés : l'aponévrose temporale, la sciure d'os, la colle biologique, le greffon de corticale osseuse, l'hydroxyapatite. Les suites opératoires sont marquées par une amélioration de la symptomatologie vestibulaire alors que l'audition reste en général inchangée. Des cas d'altération de la conduction osseuse post opératoire ont été rapportés justifiant l'abstention en cas de pathologie peu invalidante $(7,8)$.

\section{CONCLUSION}

Toute surdité de transmission ou mixte à tympan normal doit faire évoquer une déhiscence du canal semi circulaire supérieur, bien que la symptomatologie la plus typique consiste en des vertiges apparaissant lors de l'exposition à des sons de forte intensité ou lors de variations pressionnelles. Le diagnostic est confirmé par un scanner de rocher de haute résolution en coupes coronale, axiale et par des reconstructions dans l'axe du canal semi circulaire supérieur.

Le traitement chirurgical n'est indiqué qu'en cas de vertiges invalidants.

\section{REFERENCES}

1. Minor L.B, D. Solomon, J.S. Zinreich, D.S. Zee. Sound- and/ or pressure-induced vertigo due to bone dehiscence of the superior semicircular canal. Arch. Otolaryngol. Head Neck Surg 1998; 3: 249 - 258.

2. Minor LB, Lloyd MD. Clinical manifestations of superior semicircular canal dehiscence. Laryngoscope 2005; 115: 1717-1727.

3. Carey JP, Minor LB, Nager GT. Dehiscence or thinning of bone overlying the superior semicircular canal in a temporal bone survey. Arch Otolaryngol Head Neck Surg 2000; 126: 137-147.

4. Mikulec AA, McKenna MJ, Ramsey MJ et al. Superior semicircular canal dehiscence presenting as conductive hearing loss without vertigo. Otol Neurotol 2004; 25: 121-129.

5. Brantberg K, Bergenius J, Mendel L, Witt H, Tribukait A, Ygge J. Symptoms, findings and treatment in patients with dehiscence of the superior semicircular canal. Acta Otolaryngol 2001; 121: 68-75.

6 . Streubel SO, Cremer PD, Carey JP, Weg N, Minor LB. Vestibular-evoked myogenic potentials in the diagnosis of superior canal dehiscence syndrome. Acta Otolaryngol 2001; 545: 41-49.

7. Ramsey MJ, McKenna MJ, Barker FG 2nd. Superior semicircular canal dehiscence syndrome. Case report. J Neurosurg 2004; 100: 123-124.

8. Minor LB. Superior canal dehiscence syndrome. Am J Otol 2000; 21: 9-19.

9. Williamson RA, Vrabec JT, Coker NJ, Sandlin M. Coronal computed tomography prevalence of superior semicircular canal dehiscence.
Otolaryngol Head Neck Surg 2003; 129: 481-489.

10. Barton FB, Harrigal C, Hirsch BE, Superior semicircular canal dehiscence: oblique reformatted CT images for diagnosis. Radiology 2006; 238: 938-942.

11. Rosowski JJ, Songer JE, Nakajima HH, Brinsko KM, Merchant SN. Clinical, experimental and theoretical investigations of the effect of superior semicircular canal dehiscence on hearing mechanisms. Otol Neurotol 2004; 25: 323-332

12. Cox KM, Lee DJ, Carey JP, Minor LB. Dehiscence of bone overlying the superior semicircular canal as a cause of an air-bone gap on audiometry: a case study. Am J Audiol 2003; 12: 11-16.

13. Hirvonen TP, Carey JP, Liang CJ, Minor LB. Superior canal dehiscence: mechanisms of pressure sensitivity in a chinchilla model. Arch Otolaryngol Head Neck Surg 2001; 127: 1331-1336.

14. Carey JP, Hirvonen TP, Hullar TE, Minor LB. Acoustic responses of vestibular afferents in a model of superior canal dehiscence. Otol Neurotol 2004; 25: 345-352.2

15. Crovetto M., Areitio E, Elexpuru J, Aguayo F. Transmastoid approach for resurfacing of superior semicircular canal dehiscence. Auris Nasus Larynx 2008; 35: 247-249. 